\title{
Various Heteroclinic Solutions for the Coupled Schrödinger-Boussinesq Equation
}

\author{
Murong Jiang' and Zhengde Dai ${ }^{2}$ \\ ${ }^{1}$ School of Information Science and Engineering, Yunnan University, Kunming 650091, China \\ ${ }^{2}$ School of Mathematics and Statistics, Yunnan University, Kunming 650091, China
}

Correspondence should be addressed to Zhengde Dai; zhddai2004@yahoo.com.cn

Received 31 December 2012; Accepted 21 February 2013

Academic Editor: Peicheng Zhu

Copyright (c) 2013 M. Jiang and Z. Dai. This is an open access article distributed under the Creative Commons Attribution License, which permits unrestricted use, distribution, and reproduction in any medium, provided the original work is properly cited.

Various closed-form heteroclinic breather solutions including classical heteroclinic, heteroclinic breather and Akhmediev breathers solutions for coupled Schrödinger-Boussinesq equation are obtained using two-soliton and homoclinic test methods, respectively. Moreover, various heteroclinic structures of waves are investigated.

\section{Introduction}

The existence of the homoclinic and heteroclinic orbits is very important for investigating the spatiotemporal chaotic behavior of the nonlinear evolution equations (NEEs). In recent years, exact homoclinic and heterclinic solutions were proposed for some NEEs like nonlinear Schrödinger equation, Sine-Gordon equation, Davey-Stewartson equation, Zakharov equation, and Boussinesq equation [1-7].

The coupled Schrödinger-Boussinesq equation is considered as

$$
\begin{gathered}
i E_{t}+E_{x x}+\beta_{1} E-N E=0, \\
3 N_{t t}-N_{x x x x}+3\left(N^{2}\right)_{x x}+\beta_{2} N_{x x}-\left(|E|^{2}\right)_{x x}=0,
\end{gathered}
$$

with the periodic boundary condition

$$
E(x, t)=E(x+l, t), \quad N(x, t)=N(x+l, t),
$$

where $l, \beta_{1}, \beta_{2}$ are real constants, $E(x, t)$ is a complex function, and $N(x, t)$ is a real function. Equation (1) has also appeared in [8] as a special case of general systems governing the stationary propagation of coupled nonlinear upperhybrid and magnetosonic waves in magnetized plasma. The complete integrability of (1) was studied by Chowdhury et al.
[9], and $N$-soliton solution, homoclinic orbit solution, and rogue solution were obtained by $\mathrm{Hu}$ et al. [10], Dai et al. [1113], and $\mathrm{Mu}$ and Qin [14].

\section{Linear Stability Analysis}

It is easy to see that $\left(e^{i \theta_{0}}, \beta_{1}\right)$ is a fixed point of (1), and $\theta_{0}$ is an arbitrary constant. We consider a small perturbation of the form

$$
E=e^{i \theta_{0}}(1+\epsilon), \quad N=\beta_{1}(1+\phi),
$$

where $|\epsilon(x, t)| \ll 1,|\phi(x, t)| \ll 1$. Substituting (3) into (1), we get the linearized equations

$$
i \epsilon_{t}+\epsilon_{x x}-\beta_{1} \phi=0
$$

$$
3 \phi_{t t}-\phi_{x x x x}+\left(\beta_{2}+2 \beta_{1}^{2}\right) \phi_{x x}-\epsilon_{x x}-\bar{\epsilon}_{x x}=0 .
$$

Assume that $\epsilon$ and $\phi$ have the following forms:

$$
\begin{aligned}
\epsilon & =G e^{i \mu_{n} x+\sigma_{n} t}+H e^{-i \mu_{n} x+\sigma_{n} t}, \\
\phi & =C\left(e^{i \mu_{n} x+\sigma_{n} t}+e^{-i \mu_{n} x+\sigma_{n} t}\right),
\end{aligned}
$$

where $G, H$ are complex constants, and $C$ is a real number; $\mu_{n}=2 \pi n / l$, and $\sigma_{n}$ is the growth rate of the $n$th modes. 
Substituting (5) into (4), we have

$$
\begin{gathered}
G\left(i \sigma_{n}-\mu_{n}^{2}\right)=\beta_{1} C, \\
H\left(i \sigma_{n}-\mu_{n}^{2}\right)=\beta_{1} C, \\
\left(3 \sigma_{n}^{2}-\mu_{n}^{4}-\mu_{n}^{2}\left(\beta_{2}+2 \beta_{1}^{2}\right)\right) C=-(G+\bar{H}) v_{n}^{2}, \\
\left(3 \sigma_{n}^{2}-\mu_{n}^{4}-\mu_{n}^{2}\left(\beta_{2}+2 \beta_{1}^{2}\right)\right) C=-(H+\bar{G}) \mu_{n}^{2} .
\end{gathered}
$$

Solving (6), we obtain that

$$
\sigma_{n}^{2}=\frac{\mu_{n}^{2}\left(\beta_{2}+2 \beta_{1}^{2}\right)-2 \mu_{n}^{4} \pm \sqrt{\Delta}}{6}
$$

with

$$
\begin{aligned}
\Delta= & 4 \mu_{n}^{8}+\mu_{n}^{4}\left(\beta_{2}+2 \beta_{1}^{2}\right)^{2}-4 \mu_{n}^{6}\left(\beta_{2}+2 \beta_{1}^{2}\right) \\
& +12 \mu_{n}^{4}\left(\mu_{n}^{4}+\mu_{n}^{2}\left(\beta_{2}+2 \beta_{1}^{2}\right)-2 \beta_{1}\right) .
\end{aligned}
$$

Obviously, (7) implies that $\mu_{n}^{2}\left(\beta_{2}+2 \beta_{1}^{2}\right)-2 \mu_{n}^{4}>0$; then,

$$
\mu_{n}^{2}<\frac{\beta_{2}+2 \beta_{1}^{2}}{2}
$$

\section{Various Heterclinic Breather Solutions}

Set

$$
E(x, t)=e^{-i a t} u(x, t), \quad N(x, t)=v_{0}+v(x, t) .
$$

Substituting (10) into (1), we get

$$
\begin{gathered}
i u_{t}+u_{x x}+\left(a+\beta_{1}-v_{0}\right) u=u v \\
3 v_{t t}-v_{x x x x}+\left(6 v_{0}+\beta_{2}\right) v_{x x}+3\left(v^{2}\right)_{x} x=\left(|u|^{2}\right)_{x x} .
\end{gathered}
$$

We can choose $a, v_{0}$ such that $a+\beta_{1}-v_{0}=0$.

By using the following transformation

$$
u=\frac{g(x, t)}{f(x, t)}, \quad v=-2(\ln f(x, t))_{x x} .
$$

Equation (11) can be reduced into the following bilinear form:

$$
\begin{gathered}
\left(i D_{t}+D_{x}^{2}\right) g \cdot f=0, \\
\left(3 D_{t}^{2}+\left(6 v_{0}+\beta_{2}\right) D_{x}^{2}-D_{x}^{4}-\lambda\right) f \cdot f+g g^{*}=0,
\end{gathered}
$$

where $g(x, t)$ is an unknown complex function and $f(x, t)$ is a real function, $g^{*}$ is conjugate function of $g(x, t)$, and $\lambda$ is an integration constant. The Hirota bilinear operators $D_{x}^{m} D_{t}^{n}$ are defined by

$$
\begin{aligned}
D_{x}^{m} & D_{t}^{n} f(x, t) \cdot g(x, t) \\
& =\left(\frac{\partial}{\partial x}-\frac{\partial}{\partial x^{\prime}}\right)^{m}\left(\frac{\partial}{\partial t}-\frac{\partial}{\partial t^{\prime}}\right)^{n}\left[f(x, t) g\left(x^{\prime}, t^{\prime}\right)\right]_{x^{\prime}=x, t^{\prime}=t} .
\end{aligned}
$$

We use three test functions to investigate the variation of the heterclinic solution for the coupled SchrödingerBoussinesq equation (1). (1) We seek the following forms of the heterclinic solution:

$$
\begin{aligned}
& g=1+b_{1} \cos (p x) e^{\Omega t+\gamma}+b_{2} e^{2 \Omega t+2 \gamma}, \\
& f=1+b_{3} \cos (p x) e^{\Omega t+\gamma}+b_{4} e^{2 \Omega t+2 \gamma},
\end{aligned}
$$

where $b_{1}, b_{2}$ are complex numbers and $b_{3}, b_{4}$ are real numbers. $b_{i}(i=1,2,3,4), p, \Omega, \gamma$ will be determined later.

Choosing $v_{0}=\beta_{1}$, then $a=0$. Substituting (15) into the (13), we have the following relations among these constants:

$$
\begin{gathered}
\lambda=1, \quad b_{1}=\frac{i \Omega+p^{2}}{i \Omega-p^{2}} b_{3}, \\
b_{2}=\left(\frac{i \Omega+p^{2}}{i \Omega-p^{2}}\right)^{2} b_{4}, \quad b_{4}=\frac{\Omega^{2}+p^{4}}{4 \Omega^{2}} b_{3}^{2}, \\
\left(3 \Omega^{2}-p^{4}-\left(6 \beta_{1}+\beta_{2}\right) p^{2}\right)\left(\Omega^{2}+p^{4}\right)=2 p^{4} .
\end{gathered}
$$

Therefore, we have the heterclinic solution for (1) as:

$$
E(x, t)=\frac{e^{\Omega t+\gamma}+b_{1} \cos (p x)+b_{2} e^{\Omega t+\gamma}}{\sqrt{b_{4}}\left(2 \cosh \left(\Omega t+\gamma+\ln \sqrt{b_{4}}\right)+b_{3} \cos (p x)\right)},
$$

$N(x, t)$

$$
=\beta_{1}+\frac{2 b_{3} p^{2}\left(2 \sqrt{b_{4}} \cos (p x) \cosh \left(\Omega t+\gamma+\ln \sqrt{b_{4}}\right)+b_{3}\right)}{b_{4}\left(2 \cosh \left(\Omega t+\gamma+\ln \sqrt{b_{4}}\right)+b_{3} \cos (p x)\right)^{2}} .
$$

It is easy to see that $(E, N) \rightarrow\left(1, \beta_{1}\right)$ as $t \rightarrow-\infty$ and $(E, N) \rightarrow\left(\left(\left(i \Omega+p^{2}\right) /\left(i \Omega-p^{2}\right)\right)^{2}, \beta_{1}\right)$ as $t \rightarrow+\infty$. After giving some constants in (17), we find that the shape of the heterclinic orbit for Schrödinger-Boussinesq equation likes the hook, and the orbits are heterclinic to two different fixed points (see Figure 1 with $\beta_{1}=1, \beta_{2}=-2, p=1$, and $\gamma=1$ ).

(2) We take ansatz of extended homoclinic test approach for (13) as follows:

$$
\begin{aligned}
f(x, t)= & e^{-p_{1}(x-\alpha t)-\eta_{0}}+b_{3} \cos \left(p(x+\alpha t)+\eta_{1}\right) \\
& +b_{4} e^{p_{1}(x-\alpha t)+\eta_{0}}, \\
g(x, t)= & e^{-i \theta}\left(e^{-p_{1}(x-\alpha t)-\eta_{0}}+b_{1} \cos \left(p(x+\alpha t)+\eta_{1}\right)\right. \\
& \left.+b_{2} e^{p_{1}(x-\alpha t)+\eta_{0}}\right),
\end{aligned}
$$

where the parameters $p, p_{1}, \alpha, \eta_{0}, \eta_{1}, b_{s}(s=1,2,3,4)$ will be determined later, $b_{1}$ and $b_{2}$ are complex numbers, and $b_{3}$ and $b_{4}$ are real numbers. Substituting (18) into (13) and choosing $v_{0}=\beta_{1}$, we get the following relations among the parameters: 


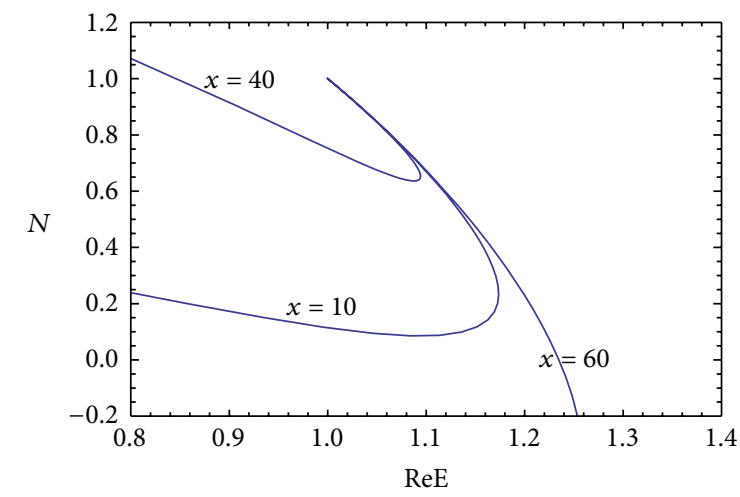

(a)

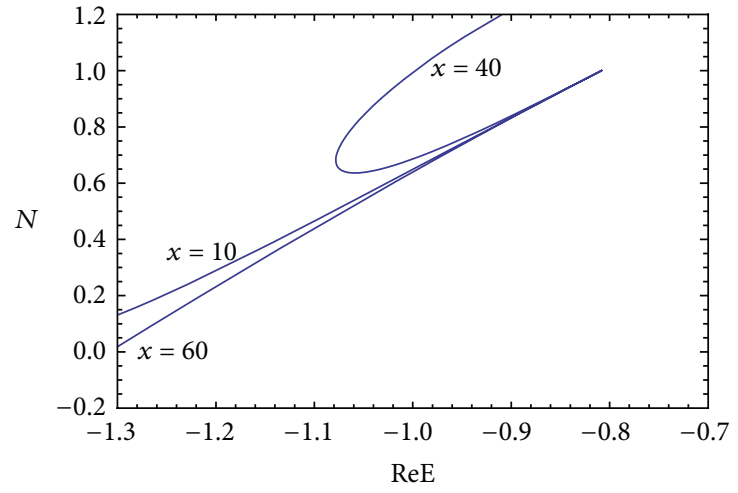

(b)

Figure 1: Hook heteroclinic orbits for Schrödinger-Boussinesq equation as $t \rightarrow-\infty$ (a) and $t \rightarrow+\infty$ (b).

$$
\begin{gathered}
p^{2}=3 p_{1}^{2}, \quad \lambda=1, \\
p_{1}^{2}=\frac{3}{4} \alpha^{2}-\frac{1}{4} \beta_{2}-\frac{3}{2} \beta_{1}, \quad \alpha^{2}=\frac{\left(\beta_{2}+6 \beta_{1}\right)^{2}-2}{4\left(\beta_{2}+6 \beta_{1}\right)}, \\
b_{1}=\frac{b_{3}\left(i \alpha-2 p_{1}\right)}{i \alpha+2 p_{1}}, \quad b_{2}=\frac{b_{4}\left(i \alpha-2 p_{1}\right)^{2}}{\left(i \alpha+2 p_{1}\right)^{2}}, \\
b_{3}= \pm \frac{2 p_{1} \sqrt{\left(3 \alpha^{2}-4 p_{1}^{2}\right) b_{4}}}{p \sqrt{\alpha^{2}+4 p_{1}^{2}}} .
\end{gathered}
$$

From (19), we get the restrictive conditions with

$$
-\sqrt{2}<\beta_{2}+6 \beta_{1}<0, \quad b_{4}<0 .
$$

Denote that $\left(i \alpha-2 p_{1}\right) /\left(i \alpha+2 p_{1}\right)=e^{i \theta_{0}}$. Then, substituting (10) into (1) and employing (19), we obtain the solution of the coupled Schrödinger-Boussinesq equation as follows:

$$
\begin{gathered}
E(x, t)=e^{i\left(\theta_{0}-\theta\right)} \frac{2 \sqrt{-b_{4}} \sinh \left(p_{1}(x-\alpha t)+\eta_{0}+\ln \left(\sqrt{-b_{4}}\right)+i \theta_{0}\right)-b_{3} \cos \left(p(x+\alpha t)+\eta_{1}\right)}{2 \sqrt{-b_{4}} \sinh \left(p_{1}(x-\alpha t)+\eta_{0}+\ln \left(\sqrt{-b_{4}}\right)\right)-b_{3} \cos \left(p(x+\alpha t)+\eta_{1}\right)}, \\
N(x, t)=\beta_{1}-\frac{8 \sqrt{-b_{4}} b_{3} p_{1}^{2} \sinh \left(p_{1}(x-\alpha t)+\eta_{0}+\ln \left(\sqrt{-b_{4}}\right)\right) \cos \left(p(x+\alpha t)+\eta_{1}\right)}{\left(2 \sqrt{-b_{4}} \sinh \left(p_{1}(x-\alpha t)+\eta_{0}+\ln \left(\sqrt{-b_{4}}\right)\right)-b_{3} \cos \left(p(x+\alpha t)+\eta_{1}\right)\right)^{2}} \\
-\frac{2\left(-4 \sqrt{-b_{4}} p p_{1} b_{3} \cosh \left(p_{1}(x-\alpha t)+\eta_{0}+\ln \sqrt{-b_{4}}\right) \sin \left(p(x+\alpha t)+\eta_{1}\right)+\left(4 b_{4}-3 b_{3}^{2}\right) p_{1}^{2}\right)}{\left(2 \sqrt{-b_{4}} \sinh \left(p_{1}(x-\alpha t)+\eta_{0}+\ln \left(\sqrt{-b_{4}}\right)\right)-b_{3} \cos \left(p(x+\alpha t)+\eta_{1}\right)\right)^{2}},
\end{gathered}
$$

where $\eta_{0}, \eta_{1}$ are arbitrary numbers.

Solution in (21) is a heteroclinic breather wave solution. It is easy to see that $(E, N) \rightarrow\left(e^{-i\left(\theta+2 \theta_{0}\right)}, \beta_{1}\right)$ as $t \rightarrow-\infty$ and $(E, N) \rightarrow\left(e^{-i \theta}, \beta_{1}\right)$ as $t \rightarrow+\infty$. Given some constants in (21), this kind of the heterclinic orbit likes a spiral, and it is heterclinic to the points $\left(e^{-i\left(\theta+2 \theta_{0}\right)}, \beta_{1}\right)$ and $\left(e^{-i \theta}, \beta_{1}\right)$ (see Figure 2 with $\beta_{1}=-1.5, \beta_{2}=8$, and $\left.b_{4}=-4\right)$.

Note that $\left(e^{-i\left(\theta+2 \theta_{0}\right)}, \beta_{1}\right)$ and $\left(e^{-i \theta}, \beta_{1}\right)$ are two different fixed points of (21), which is a heteroclinic solution (see Figure 3). This wave also contains the periodic wave, and its amplitude periodically oscillates with the evolution of time, which shows that this wave has breather effect. The previous results combined with (21) show that interaction between a solitary wave and a periodic wave with the same velocity $\alpha$ and opposite propagation direction can form a heteroclinic breather flow. This is a new phenomenon of physics in the stationary propagation of coupled nonlinear upper-hybrid and magnetosonic waves in magnetized plasma.

(3) Use the following forms of the heterclinic solution [14]:

$$
\begin{gathered}
g=b_{1} \cosh (\alpha t)+b_{2} \cos (p x)+b_{3} \sinh (\alpha t), \\
f=b_{4} \cosh (\alpha t)+b_{5} \cos (p x),
\end{gathered}
$$

where $b_{1}, b_{2}, b_{3}$ are complex numbers and $b_{4}, b_{5}$ are real numbers. $b_{i}(i=1,2,3,4,5), p, \alpha$ will be determined later. 


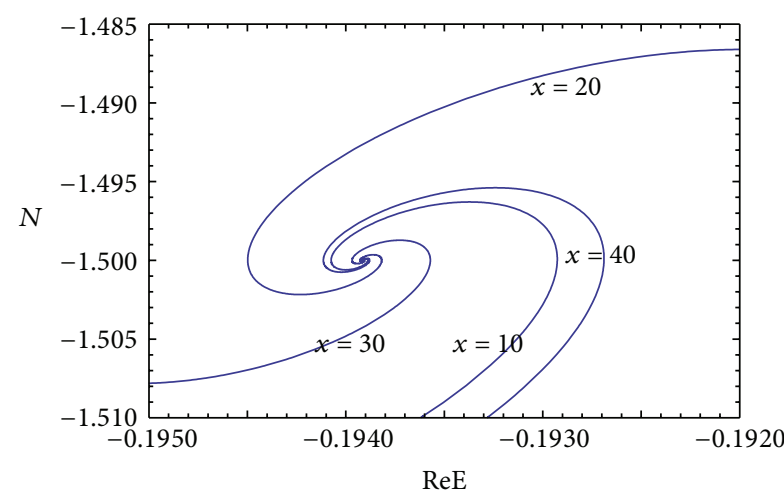

(a)

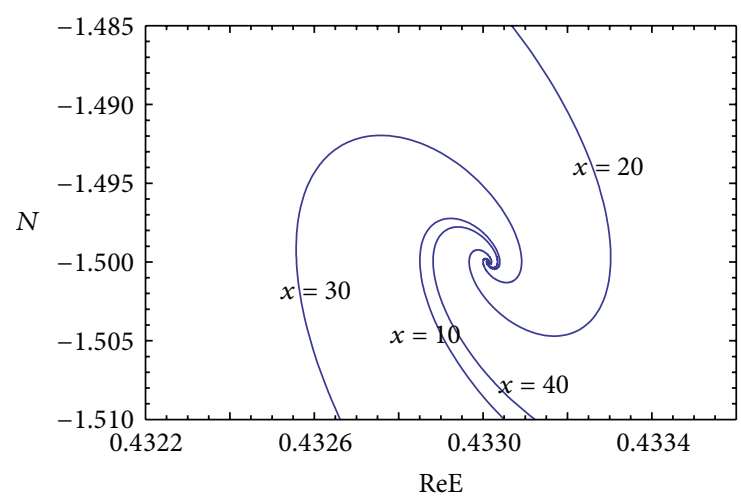

(b)

FIGURE 2: Spiral heteroclinic orbits for Schrödinger-Boussinesq equation as $t \rightarrow-\infty$ (a) and $t \rightarrow+\infty$ (b).

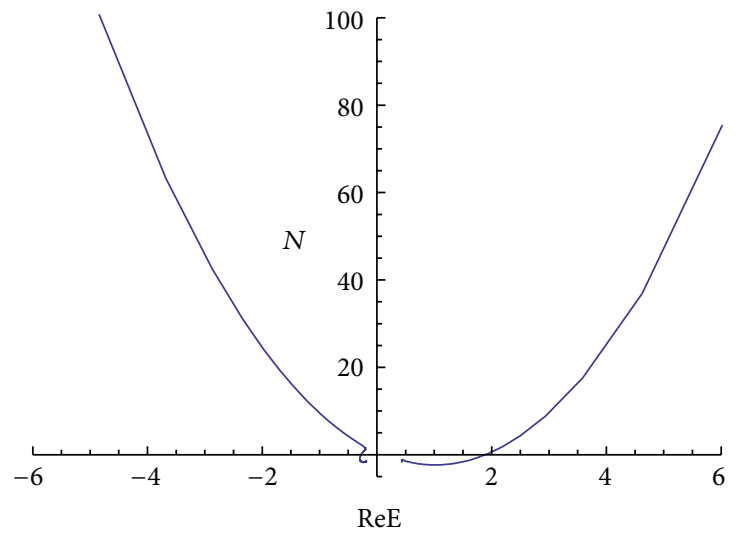

FIGURE 3: One heteroclinic orbit for Schrödinger-Boussinesq equation as $x=0$.

We also choose $v_{0}=\beta_{1}$ and substitute (22) into (13). We have the following relations among these constants:

$$
\begin{gathered}
i b_{3} b_{4} \alpha=b_{2} b_{5} p^{2}, \\
b_{5}\left(b_{1}+b_{3}\right)\left(i \alpha-p^{2}\right)=b_{2} b_{4}\left(i \alpha+p^{2}\right), \\
b_{2} b_{4}\left(i \alpha-p^{2}\right)=b_{5}\left(b_{1}-b_{3}\right)\left(i \alpha+p^{2}\right), \\
-b_{4}^{2}+12 \alpha^{2} b_{4}^{2}-2 b_{5}^{2} \cos ^{2}(p x)-16 b_{5}^{2} p^{4}-4 b_{5}^{2} p^{2}\left(6 \beta_{1}+\beta_{2}\right) \\
+b_{1} b_{1}^{*}-b_{3} b_{3}^{*}+2 b_{2} b_{2}^{*} \cos ^{2}(p x)=0 .
\end{gathered}
$$

Solving (23), we get

$$
\begin{gathered}
b_{1}=\frac{\left(p^{4}-\alpha^{2}\right) b_{2}}{\alpha \sqrt{2\left(\alpha^{2}+p^{4}\right)}}, \quad b_{3}= \pm i \frac{\sqrt{2} p^{2} b_{2}}{\sqrt{\alpha^{2}+p^{4}}}, \\
b_{4}^{2}=\frac{\left(\alpha^{2}+p^{4}\right) b_{5}^{2}}{2 \alpha^{2}} .
\end{gathered}
$$

Therefore, we have the heterclinic solution for (1) as

$$
\begin{aligned}
& E(x, t)=\frac{b_{1} \cosh (\alpha t)+b_{2} \cos (p x)+b_{3} \sinh (\alpha t)}{b_{4} \cosh (\alpha t)+b_{5} \cos (p x)}, \\
& N(x, t)=\beta_{1}+2 \frac{b_{5} p^{2}\left(b_{4} \cos (p x) \cosh (\alpha t)+b_{5}\right)}{\left(b_{4} \cosh (\alpha t)+b_{5} \cos (p x)\right)^{2}} .
\end{aligned}
$$

Giving some special parameters in (25), we see that the shape of the heterclinic orbits likes the arc (see Figure 4 with $\beta_{1}=1$, $\alpha=\sqrt{3}$, and $p=\sqrt{2})$. The fixed points are $(E, N) \rightarrow\left(\left(b_{1}-\right.\right.$ $\left.\left.b_{3}\right) / b_{4}, \beta_{1}\right)$ as $t \rightarrow-\infty$ and $(E, N) \rightarrow\left(\left(b_{1}+b_{3}\right) / b_{4}, \beta_{1}\right)$ as $t \rightarrow+\infty$.

\section{Conclusion}

In this work, by using three special test functions in twosoliton method and homoclinic test method, we obtain three families of heteroclinic breather wave solution heteroclinic to two different fixed points, respectively. Moreover, we investigate different structures of these wave solutions. These results show that the Schrödinger-Boussinesq equation has the variety of heteroclinic structure. As the further work, we 


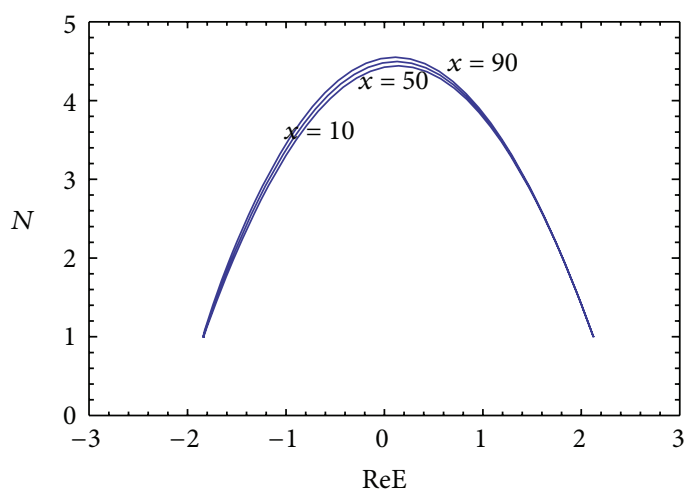

(a)

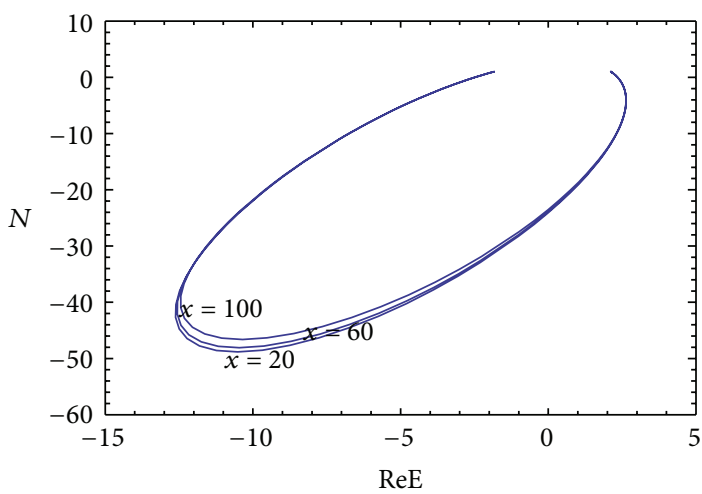

(b)

Figure 4: Arc Heteroclinic orbit for Schrödinger-Boussinesq equation as $t \rightarrow \pm \infty$ at $x=10 *(2 k+1)$ (a) and $x=10 *(4 k+2)(b)$, where $k=0,1,2, \ldots$.

will consider whether there exist the spatiotemporal chaos for the coupled Schrödinger-Boussinesq equation or not.

\section{Acknowledgments}

This work was supported by Chinese Natural Science Foundation Grant nos. 11161055 and 11061028, as well as Yunnan NSF Grant no. 2008PY034.

\section{References}

[1] M. J. Ablowitz and B. M. Herbst, "On homoclinic structure and numerically induced chaos for the nonlinear Schrödinger equation," SIAM Journal on Applied Mathematics, vol. 50, no. 2, pp. 339-345, 1990.

[2] N. Ercolani, M. G. Forest, and D. W. McLaughlin, "Geometry of the modulational instability. III. Homoclinic orbits for the periodic sine-Gordon equation," Physica D, vol. 43, no. 2-3, pp. 349-384, 1990.

[3] Z. Dai and J. Huang, "Homoclinic tubes for the DaveyStewartson II equation with periodic boundary conditions," Chinese Journal of Physics, vol. 43, no. 2, pp. 349-360, 2005.

[4] Z. Dai, J. Huang, M. Jiang, and S. Wang, "Homoclinic orbits and periodic solitons for Boussinesq equation with even constraint," Chaos, Solitons and Fractals, vol. 26, no. 4, pp. 1189-1194, 2005.

[5] Z. Dai, J. Huang, and M. Jiang, "Explicit homoclinic tube solutions and chaos for Zakharov system with periodic boundary," Physics Letters A, vol. 352, no. 4-5, pp. 411-415, 2006.

[6] N. N. Rao, "Near-magnetosonic envelope upper-hybrid waves," Journal of Plasma Physics, vol. 39, no. 3, pp. 385-392, 1988.

[7] Y. Hase and J. Satsuma, "An N-soliton solution for the nonlinear Schrödinger equation coupled to the Boussinesq equation," Journal of the Physical Society of Japan, vol. 57, no. 3, pp. 679$682,1988$.

[8] R. Conte and M. Mussete, "Link between solitary waves and Riccati equation," Journal of Physics A, vol. 25, pp. 5600-5615, 1992.

[9] A. R. Chowdhury, B. Dasgupta, and N. N. Rao, "Painléve analysis and Backlund transformations for coupled generalized Schrödinger-Boussinesq system," Chaos, Solitons and Fractals, vol. 9, no. 10, pp. 1747-1753, 1998.
[10] X. B. Hu, B. L. Guo, and H. W. Tam, "Homoclinic orbits for the coupled Schrödinger-Boussinesq equation and coupled higgs equation," Journal of the Physical Society of Japan, vol. 72, no. 1, pp. 189-190, 2003.

[11] Z. D. Dai, Z. J. Liu, and D. L. Li, "Exact periodic solitary-wave solution for KdV equation," Chinese Physics Letters, vol. 25, no. 5, pp. 1531-1534, 2008.

[12] Z. Dai, J. Liu, and D. Li, "Applications of HTA and EHTA to YTSF equation," Applied Mathematics and Computation, vol. 207, no. 2, pp. 360-364, 2009.

[13] Z. Dai, Z. Li, Z. Liu, and D. Li, "Exact homoclinic wave and soliton solutions for the 2D Ginzburg-Landau equation," Physics Letters A, vol. 372, no. 17, pp. 3010-3014, 2008.

[14] G. Mu and Z. Qin, "Rogue waves for the coupled SchrödingerBoussinesq equation and the coupled Higgs equation," Journal of the Physical Society of Japan, vol. 81, Article ID 084001, 2012. 


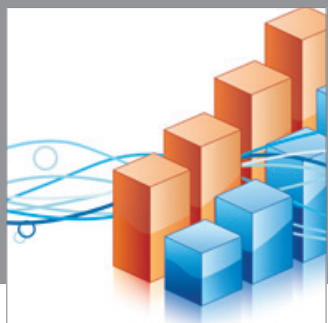

Advances in

Operations Research

mansans

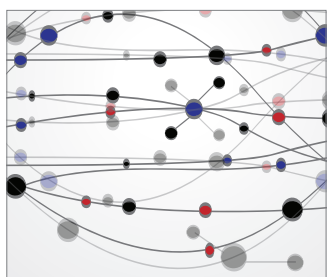

The Scientific World Journal
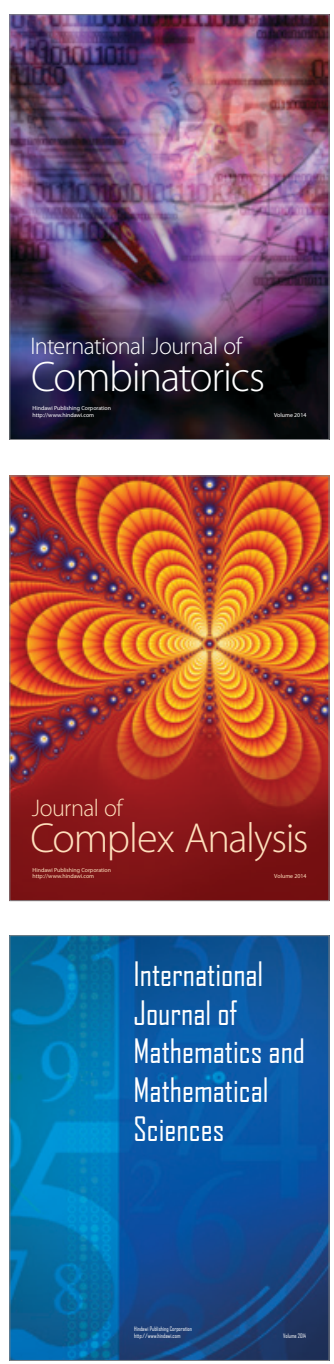
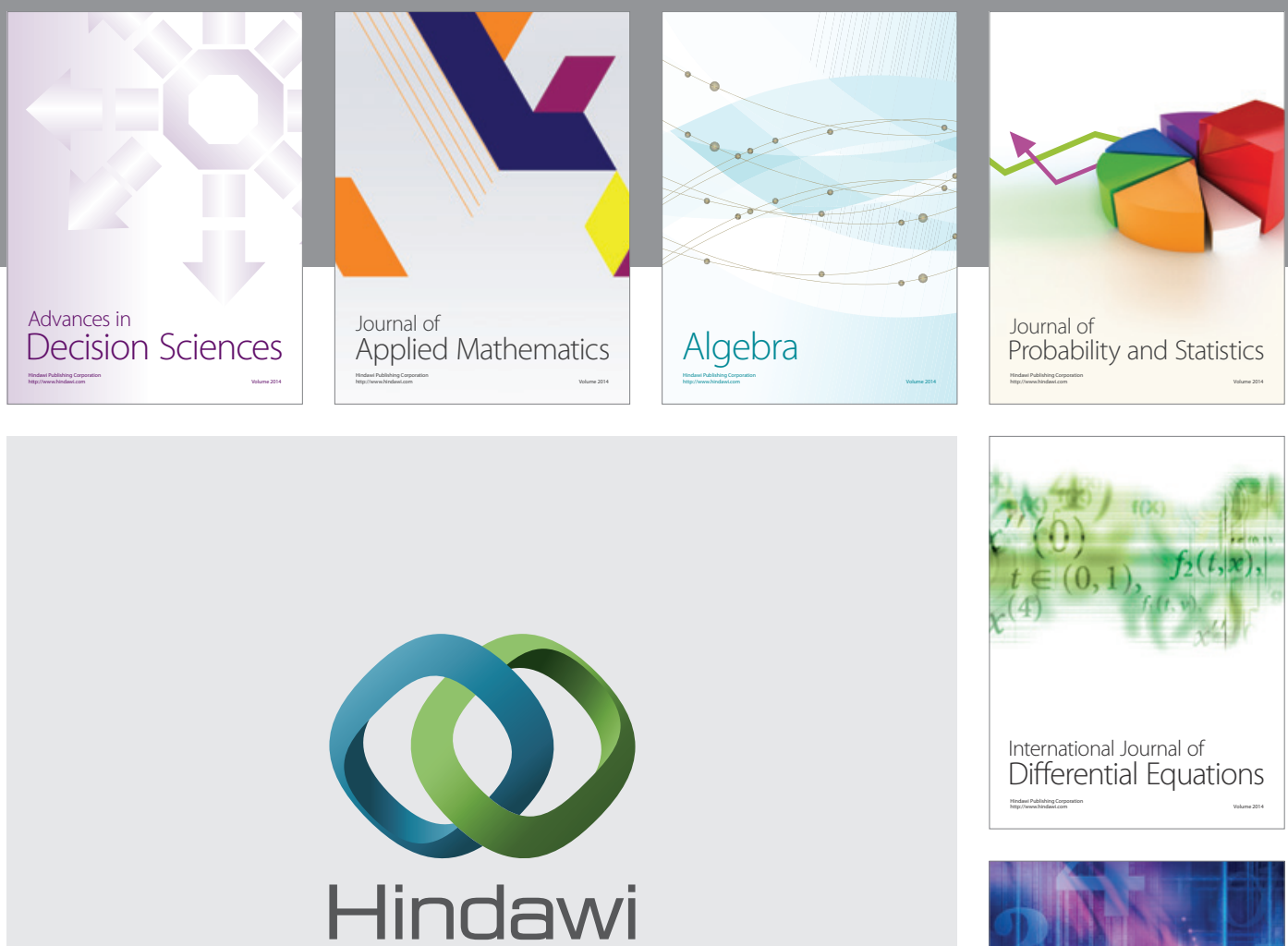

Submit your manuscripts at http://www.hindawi.com
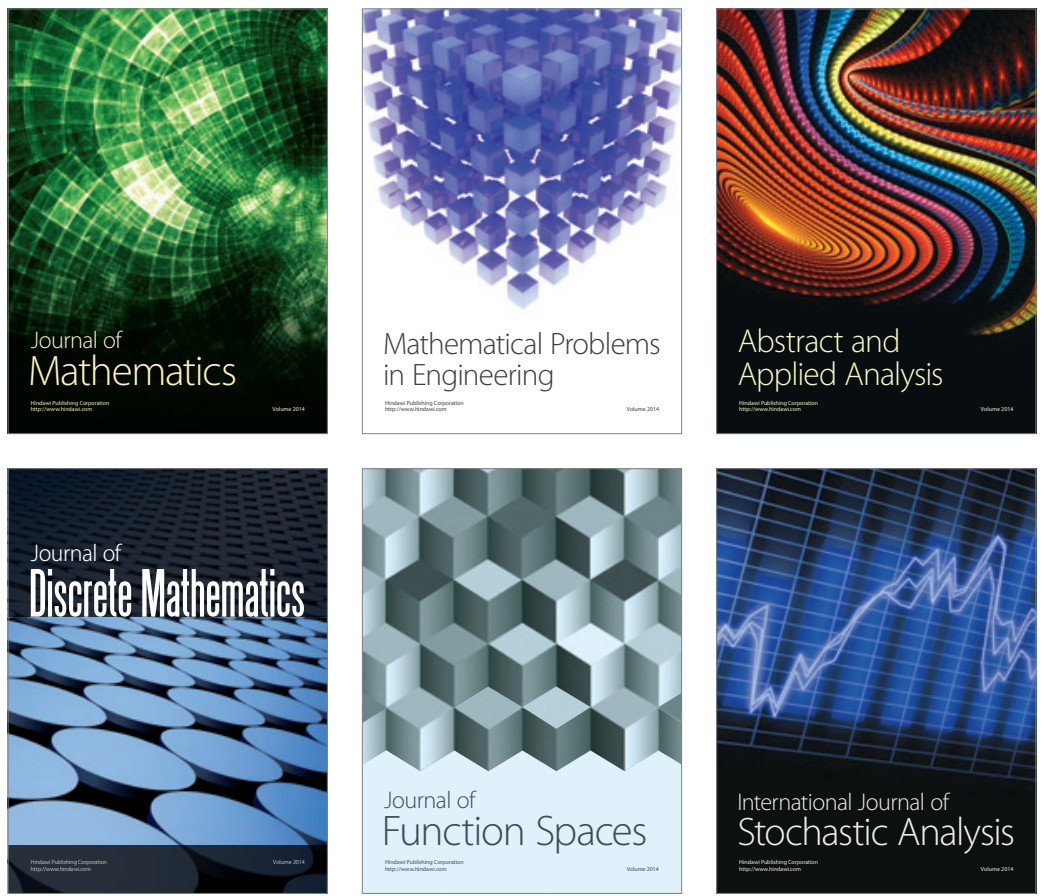

Journal of

Function Spaces

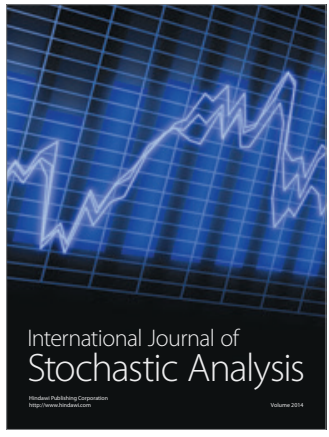

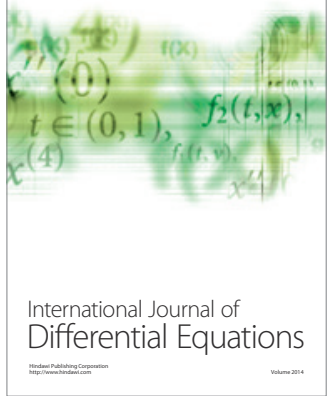
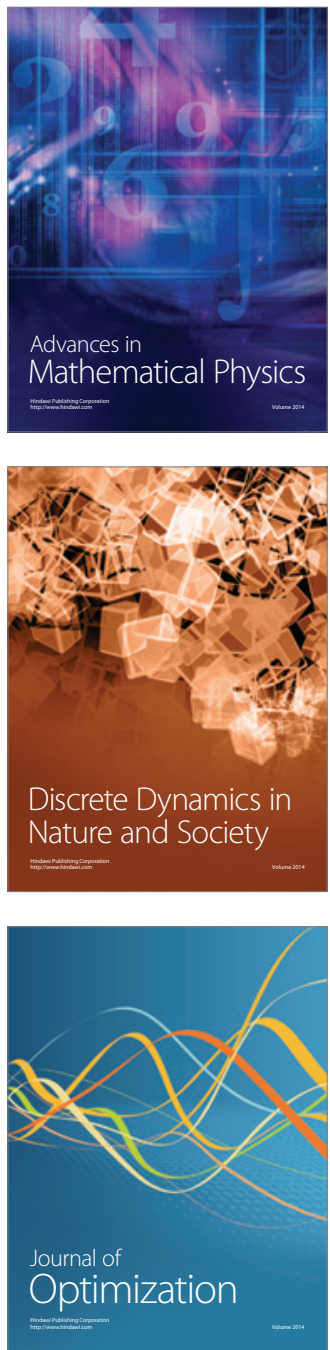